\title{
Quality Evaluation of Polar and Active Components in Crude and Processed Fructus Corni by Quantitative Analysis of Multicomponents with Single Marker
}

\author{
Yuhong Jiang, ${ }^{1}$ Hui Chen, ${ }^{1}$ Liling Wang, ${ }^{1}$ Jing Zou, ${ }^{1}$ Xiao Zheng, ${ }^{2}$ and Zhihui Liu ${ }^{2}$ \\ ${ }^{1}$ College of Pharmacy, Nanjing University of Chinese Medicine, Nanjing 210023, China \\ ${ }^{2}$ Department of Pharmacy, Nanjing University of Chinese Medicine Affiliated Hospital, Nanjing 210029, China \\ Correspondence should be addressed to Zhihui Liu; liuzh1008@126.com
}

Received 23 May 2016; Accepted 8 June 2016

Academic Editor: Sibel A. Ozkan

Copyright ( $\odot 2016$ Yuhong Jiang et al. This is an open access article distributed under the Creative Commons Attribution License, which permits unrestricted use, distribution, and reproduction in any medium, provided the original work is properly cited.

Objective. To develop a quantitative analysis of multicomponents by single-marker (QAMS) method for the simultaneous determination of polar active components in Fructus Corni. Methods. Loganin was selected as the internal reference, and the relative correction factors (RCFs) of gallic acid, 5-hydroxymethyl-2-furfural, morroniside, sweroside, cornin, $7 \alpha$-O-methylmorroniside, $7 \beta$-O-methylmorroniside, $7 \alpha$-O-ethylmorroniside, $7 \beta$-O-ethylmorroniside, and cornuside were established. The contents of multicomponents were then calculated based on their RCFs, respectively. Contents of the 11 components were also calculated by external standard method and compared with those of the QAMS method. Results. The contents of the 11 components in 21 crude and 10 processed Fructus Corni products were measured. No significant difference was found in the quantitative results of the QAMS and external standard methods. Conclusion. QAMS could serve as an accurate and convenient method in determining the polar and active components in Fructus Corni and its processed products.

\section{Introduction}

Fructus Corni is derived from the dried ripe sarcocarp of Cornus officinalis Sieb. et Zucc (Cornaceae). In the practice of traditional medicine, Fructus Corni has a role in nourishing the liver and kidney, which provides the pharmacological basis for its wide use in many herbal prescriptions [1]. In particular, wine-prepared Fructus Corni, the major processed product of crude Fructus Corni, is frequently used in traditional medicine. Modern researches have shown that Fructus Corni has many chemical constituents, such as volatile constituents, iridoids, organic acids, carbohydrates, and tannins [2]. Among them, iridoid glycosides are a family of active ingredients in Fructus Corni, which are reported to have many pharmacological activities including immune regulation, glycemic lowering, anticoagulation, antiarrhythmia, neuroprotection, and memory improving [3-5]. In addition, gallic acid and 5-hydroxymethyl-2-furfural, which belong to organic acids, are reported to contribute to the effect of
Fructus Corni in improving liver and kidney functions and related antioxidative effects $[6,7]$.

In the 2015 edition of Chinese Pharmacopoeia, only morroniside and loganin are used as indicators for quality control of Fructus Corni. Since several other iridoid compounds and components like organic acids also contribute to the integrative pharmacological effects of Fructus Corni and its processed products, quantification of two components alone may not be adequate for a comprehensive evaluation of herbal quality. To date, however, quantitative methods for the active components in Fructus Corni are largely compromised by the focus on a limited number of target components [810]. Therefore, there is a clear need for the development of a quality control method that may have more comprehensive coverage of active components from Fructus Corni.

High-performance liquid chromatography (HPLC) method is frequently used for the quality control of herbal drugs for its wide accessibility and good analytical performance. Quantitative analysis of multicomponents 
by single marker (QAMS) is a working strategy in the simultaneous determination of multiple components by HPLC. A predominant advantage of this strategy is the independence of the availability of all the target components, since their concentration could be easily calculated based on the relative correction factors (RCFs) in relation to the internal compound [11]. In this paper, we aimed to develop and validate a QAMS method for the simultaneous determination of 11 high-polarity active components from Fructus Corni. The results showed that, by choosing loganin as the internal reference substance and establishing the relative correction factors (RCFs) of other 10 components, this method proved reliable, accurate, and simple for determining those active components in Fructus Corni and its processed products.

\section{Materials and Methods}

2.1. Chemicals and Reagents. 5-Hydroxymethyl-2-furfural, morroniside, sweroside, cornin, and cornuside were purchased from Nanjing Ningqi Pharmaceutical Co. Ltd. (Nanjing, China). Gallic acid and loganin were purchased from National Institute for Food and Drug Control (Beijing, China). The purity for each reference compound was greater than $98 \%$ as validated by HPLC analysis. $7 \alpha$-O-Methylmorroniside, $7 \beta$-O-methylmorroniside, $7 \alpha$-O-ethylmorroniside, and $7 \beta$-O-ethylmorroniside were prepared by our lab and structurally characterized based on ${ }^{1} \mathrm{H}-\mathrm{NMR}$ and ${ }^{13} \mathrm{C}$-NMR spectra. Their contents were $99.7 \%$ and $99.6 \%$ as determined by HPLC analysis, respectively. Acetonitrile of HPLC grade was purchased from Merck (Darmstadt, Germany). Formic acid of analytical grade was purchased from Roe scientific Inc. (USA). A Milli-Q water (Millipore, Inc., USA) purification system was used to obtain purified water for the HPLC analysis.

2.2. Herbal Plant. Crude Fructus Corni (numbers 1-21) was purchased from Anhui Xiehecheng Pharmaceutical Preparation Co., Ltd. (Bozhou, China) (batch numbers 131219, 131222, 140212, 140218, and 140403 were collected from Hubei Province; 140301, 140305, 140401, 140403, and 140528 were from Zhejiang Province; 140102, 140406, and 130616 were from Henan Province; 131219, 140102, 140301, and 140406 were from Jiangxi Province; 130616, 140301, 140305, and 140406 were from Anhui Province). Processed Fructus Corni (numbers 22-31) were also purchased from Anhui Xiehecheng Pharmaceutical Preparation Co., Ltd. (Bozhou, China) (batch numbers 131201, 140201, and 140506 were from Anhui Province; 131211 and 140216 were from Hubei Province; 131220, 140103, 140303, and 140407 were from Jiangxi Province; 140404 was from Zhejiang Province).

2.3. Instruments and Chromatographic Conditions. Analysis was performed on three different HPLC systems: Agilent 1100 series, including vacuum degasser (G1322A), quaternary pump (G1311A), autosampler (G1316A), and a ChemStation Workstation with VWD detector; Agilent 1260 series, including DAD detector and Agilent ChemStation Workstation;
TABLE 1: Conditions of the mobile phase gradient.

\begin{tabular}{lcc}
\hline$t / \min$ & $\mathrm{A} / \%$ & $\mathrm{~B} / \%$ \\
\hline 0 & 93 & 7 \\
6 & 93 & 7 \\
8 & 92 & 8 \\
20 & 92 & 8 \\
30 & 85 & 15 \\
50 & 78 & 22 \\
\hline
\end{tabular}

Waters 2695-2996 high-performance liquid chromatography, including 2998PDA detector and Empower workstation. The chromatographic separation was performed on Elite $\mathrm{C}_{18}$ $(4.6 \mathrm{~mm} \times 250 \mathrm{~mm}, 5 \mu \mathrm{m})$, Agilent $\mathrm{C}_{18}(4.6 \mathrm{~mm} \times 250 \mathrm{~mm}$, $5 \mu \mathrm{m})$, and Welch Materials $\mathrm{C}_{18}(4.6 \mathrm{~mm} \times 250 \mathrm{~mm}, 5 \mu \mathrm{m})$.

The mobile phase was composed of $0.05 \%$ formic acidwater (A) and acetonitrile (B); the gradient program was shown in Table 1. Column temperature was maintained at $30^{\circ} \mathrm{C}$. The flow rate was kept at $1 \mathrm{~mL} \cdot \mathrm{min}^{-1}$. The wavelength UV detection was set at $240 \mathrm{~nm}$, and the injection volume was $10 \mu \mathrm{L}$. Under the above chromatographic conditions, all the components were well separated (as shown in Figure 1).

2.4. Preparation of Standard Solutions. A mixed stock solution containing reference standards was prepared by dissolving weighed accurately samples of each compound in methanol, which obtained gallic acid $124.44 \mu \mathrm{g} \cdot \mathrm{mL}^{-1}$, 5-hydroxymethyl-2-furfural $120.12 \mu \mathrm{g} \cdot \mathrm{mL}^{-1}$, morroniside $720.3 \mu \mathrm{g} \cdot \mathrm{mL}^{-1}$, sweroside $60.66 \mu \mathrm{g} \cdot \mathrm{mL}^{-1}$, cornin $22.74 \mu \mathrm{g} \cdot \mathrm{mL}^{-1}$, loganin $415.2 \mu \mathrm{g} \cdot \mathrm{mL}^{-1}, 7 \alpha$-O-methylmorroniside $40.2 \mu \mathrm{g} \cdot \mathrm{mL}^{-1}, 7 \beta$ $\mathrm{O}$-methylmorroniside $81.52 \mu \mathrm{g} \cdot \mathrm{mL}^{-1}, 7 \alpha$-O-ethylmorroniside $20.16 \mu \mathrm{g} \cdot \mathrm{mL}^{-1}, 7 \beta$-O-ethylmorroniside $20.12 \mu \mathrm{g} \cdot \mathrm{mL}^{-1}$, and cornuside $108.64 \mu \mathrm{g} \cdot \mathrm{mL}^{-1}$. The further dilution with methanol gives at least six different concentrations for the establishment of calibration curves. All stock and working solutions of standards were all prepared in dark brown bottles at $4^{\circ} \mathrm{C}$ until analysis.

2.5. Preparation of Sample Solution. The powder of Fructus Corni samples was precisely weighed $(2 \mathrm{~g})$ and immersed in $50 \mathrm{~mL}$ of methanol. Additional methanol was added to make up the loss after ultrasonic extraction for $30 \mathrm{~min}$. All solutions were filtered through $0.45 \mu \mathrm{m}$ filter membranes before being injected into the HPLC system.

\section{Results and Discussion}

\subsection{Method Validation}

3.1.1. Linearity. All the above six different concentrations of mixed standard solutions were injected $(10 \mu \mathrm{L})$ and calculated; each concentration was measured twice and took the average. The calibration curves were produced by plotting the integrated peak areas $(Y)$ versus the concentration of each component $\left(X, \mu \mathrm{g} \cdot \mathrm{mL}^{-1}\right)$ and by the linear regression 


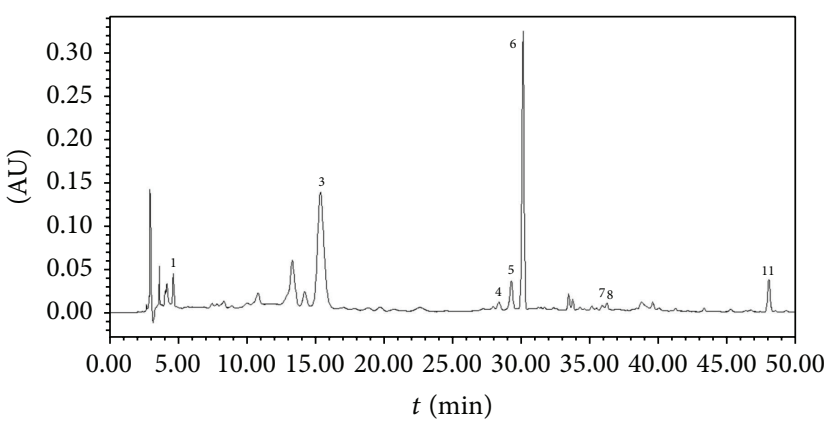

(a)

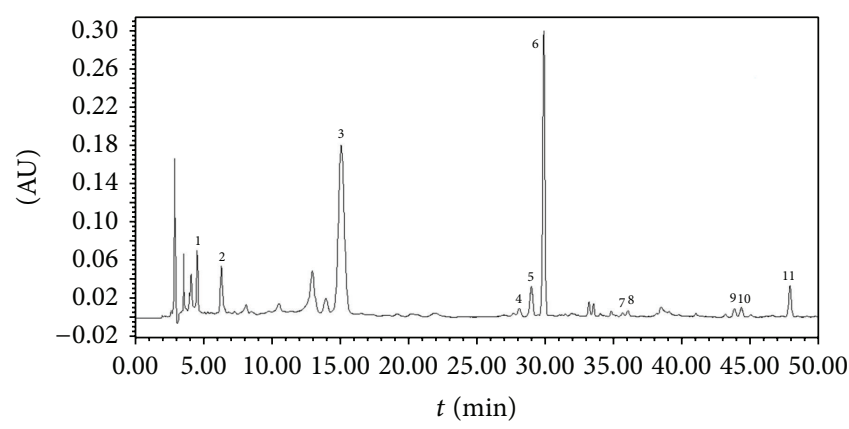

(b)

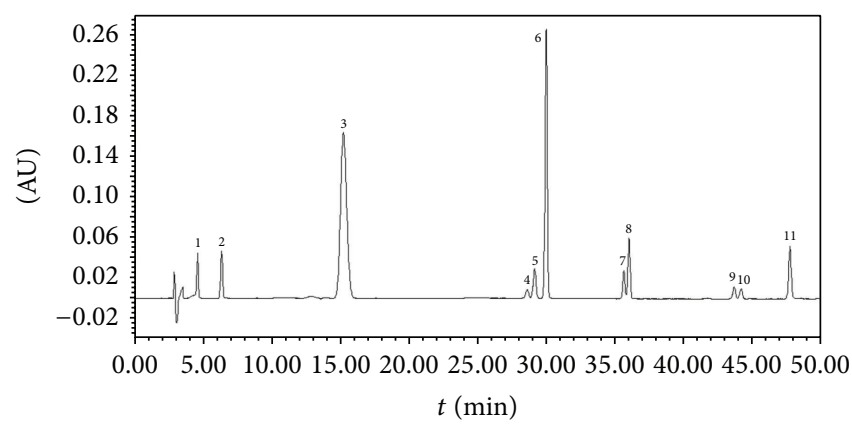

(c)

Figure 1: A chromatogram of crude Fructus Corni (a) and processed Fructus Corni (b); a chromatogram of mixture of standard compounds (c). 1: gallic acid, 2: 5-hydroxymethyl-2-furfural, 3: morroniside, 4: cornin, 5: sweroside, 6: loganin, 7: 7 $\alpha$-O-methylmorroniside, 8: 7 $\beta$-Omethylmorroniside, 9: 7 $\alpha$-O-ethylmorroniside, 10: 7 $\beta$-O-ethylmorroniside, and 11: cornuside.

TABLE 2: Standard curves of 11 components in Fructus Corni.

\begin{tabular}{|c|c|c|c|}
\hline Compound & Regression equation & $r$ & Linearity $/ \mu \mathrm{g} \cdot \mathrm{mL}^{-1}$ \\
\hline Gallic acid & $y=6411.2 x-13176$ & 0.9999 & $3.89-124.44$ \\
\hline 5-Hydroxymethylfurfural & $y=7856 x-7229$ & 0.9999 & $3.75-120.12$ \\
\hline Morroniside & $y=14534 x-64815$ & 0.9999 & $22.51-720.30$ \\
\hline Cornin & $y=10795 x-3398.3$ & 0.9995 & $0.71-22.74$ \\
\hline Sweroside & $y=13446 x-7742.1$ & 0.9998 & $1.90-60.66$ \\
\hline Loganin & $y=14317 x-32209$ & 0.9999 & $12.97-415.20$ \\
\hline $7 \alpha$-O-Methylmorroniside & $y=12640 x-3224.1$ & 0.9999 & $1.26-40.20$ \\
\hline 7 $\beta$-O-Methylmorroniside & $y=15128 x-9720.8$ & 0.9998 & $2.55-81.52$ \\
\hline $7 \alpha$-O-Ethylmorroniside & $y=14273 x-283.77$ & 0.9999 & $0.63-20.16$ \\
\hline $7 \beta$-O-Ethylmorroniside & $y=12527 x-1411.6$ & 0.9998 & $0.63-20.12$ \\
\hline Cornuside & $y=12461 x-11069$ & 0.9999 & $3.39-108.64$ \\
\hline
\end{tabular}

analysis. As was shown in Table 2, mixed standard solutions were in a certain range of linear quality.

3.1.2. Precision. The same mixed standard solution was injected six consecutive times under chromatographic conditions and their RSD values were calculated. The RSD of gallic acid, 5-hydroxymethyl-2-furfural, morroniside, cornin, sweroside, loganin, $7 \alpha$-O-methylmorroniside, $7 \beta$-O-methylmorroniside, $7 \alpha$-O-ethylmorroniside, $7 \beta$-O-ethylmorroniside, and cornuside were $0.73 \%, 1.66 \%, 1.79 \%, 2.11 \%, 1.99 \%$, $1.59 \%, 1.41 \%, 1.25 \%, 1.76 \%, 2.67 \%$, and $3.31 \%$, which indicated that the developed method had a well precision.
3.1.3. Repeatability. Six samples (processed Fructus Corni, number 23) were prepared independently for checking the repeatability. The RSD of gallic acid, 5-hydroxymethyl-2furfural, morroniside, cornin, sweroside, loganin, $7 \alpha-\mathrm{O}-$ methylmorroniside, $7 \beta$-O-methylmorroniside, $7 \alpha$-O-ethylmorroniside, $7 \beta$-O-ethylmorroniside, and cornuside were $4.05 \%, 2.60 \%, 1.60 \%, 0.97 \%, 0.93 \%, 1.43 \%, 0.68 \%, 0.69 \%$, $1.10 \%, 1.52 \%$, and $1.01 \%$. The results showed that the method had good reproducibility.

3.1.4. Stability. The stability of the same sample solution was analyzed at $0,4,8,10,12$, and $24 \mathrm{~h}$ at room temperature. 
TABLE 3: The results of recovery of eleven components in samples $(n=6)$.

\begin{tabular}{|c|c|c|c|c|c|}
\hline Compound & Original/mg & Added amount/mg & Detected amount/mg & Recovery/\% & RSD \% \\
\hline Gallic acid & 0.030 & 0.031 & 0.064 & 108.15 & 1.18 \\
\hline 5-Hydroxymethylfurfural & 0.033 & 0.033 & 0.068 & 105.29 & 1.48 \\
\hline Morroniside & 0.20 & 0.18 & 0.384 & 102.07 & 1.90 \\
\hline Cornin & 0.0017 & 0.0019 & 0.004 & 101.21 & 4.88 \\
\hline Sweroside & 0.016 & 0.017 & 0.034 & 108.73 & 1.55 \\
\hline Loganin & 0.12 & 0.114 & 0.239 & 104.27 & 0.42 \\
\hline $7 \alpha$-O-Methylmorroniside & 0.0059 & 0.0061 & 0.012 & 103.67 & 3.47 \\
\hline $7 \beta$-O-Methylmorroniside & 0.0126 & 0.013 & 0.025 & 97.25 & 1.59 \\
\hline $7 \alpha$-O-Ethylmorroniside & 0.0049 & 0.0051 & 0.010 & 95.24 & 3.40 \\
\hline $7 \beta$-O-Ethylmorroniside & 0.0053 & 0.0057 & 0.011 & 105.66 & 1.60 \\
\hline Cornuside & 0.025 & 0.024 & 0.049 & 98.54 & 0.64 \\
\hline
\end{tabular}

The RSD of gallic acid, 5-hydroxymethyl-2-furfural, morroniside, cornin, sweroside, loganin, $7 \alpha$-O-methylmorroniside, $7 \beta$-O-methylmorroniside, $7 \alpha$-O-ethylmorroniside, $7 \beta$-Oethylmorroniside, and cornuside were $1.94 \%, 3.49 \%, 0.49 \%$, $2.03 \%, 1.46 \%, 0.12 \%, 1.65 \%, 1.01 \%, 2.87 \%, 2.08 \%$, and $2.30 \%$, respectively. The results showed that the sample solutions were stable within $24 \mathrm{~h}$.

3.1.5. Recovery. Recovery test was carried out by adding reference substance solutions into a certain amount of processed Fructus Corni sample and analyzed. Six replicates were performed for the determination. The recoveries of the 11 compounds were shown in Table 3.

3.2. Calculation of Relative Correction Factors (RCFs). Loganin was selected as the internal reference substance, and the RCFs are a constant of proportionality in a computational formula and can be calculated as follows: $f_{i s}=f_{i} / f_{s}=\left(C_{i} / A_{i}\right) /\left(C_{s} / A_{s}\right)=\left(C_{i} \times A_{s}\right) /\left(C_{s} \times A_{i}\right)[12]$, where $i$ is a compound, $s$ is the internal reference substance, $C$ is the concentration of compound, and $A$ is the peak area. The RCFs of different components to loganin were calculated, respectively, and the results were shown in Table 4.

\subsubsection{Reproducibility of RCFs}

Influence of Different Instruments and Columns on RCF Values. In this paper, we injected the mixed standard solutions diluted 2, 4, and 8 times and calculated RCFs of each component. The experiment was varied on three different instruments (Waters 2695-2998, Agilent 1100, and Agilent 1260 ) and three different columns (Elite $C_{18}$, Agilent $C_{18}$, and Wel Materials $\mathrm{C}_{18}$ ); results were shown in Table 5 .

Influence of Different Columns Temperature on RCF Values. In this paper, analysis was performed on Waters 2695-2998 and Elite $\mathrm{C}_{18}$, with three different temperatures. Results were shown in Table 6.

Influence of Different Flow Rate on RCF Values. In this paper, analysis was performed on Waters 2695-2998 and Elite $C_{18}$, with three different flow rates. Results were shown in Table 7.
3.2.2. Location of Target Chromatographic Peaks. The parameter of relative retention time was used to locate the target peaks. Using loganin as the external standard, the relative retentions between the other target marker and loganin were obtained in different columns and HPLC systems. Results were shown in Table 8.

3.2.3. Comparison of the Quantitative Results between QAMS Method and External Standard Method. A total of 31 samples were injected for HPLC analysis. The contents of each component were calculated by QAMS method and external standard method; results were shown in Tables 9(a) and 9(b).

3.3. Discussion. Fructus Corni has been widely used in clinical practice for thousands of years and has well-known effects on kidney and good safety record [13]. At present, there were many reports about quantitative determination of the content of Fructus Corni and its processed products. However, most of those studies only focused on one or several few components. There were also some reports about the fingerprints of Fructus Corni, which were mainly used for qualitative analysis, but those methods could not be repeated [14-17]. In our laboratory, a method for simultaneous identification and determination of the 11 compounds in Fructus Corni using LC-MS/MS was established and validated, but LC-MS/MS method has not yet been fully popularized. In consideration of these facts, it was our striving direction to establish a quantitative analysis of multicomponents by single-marker method for the simultaneous determination of important and active components in Fructus Corni products, which was especially achievable by HPLC. In this study, we have successfully established QAMS method and realized the quantitative determination of major active components in Fructus Corni and its products.

In this paper, all the target components were iridoid glycoside components, except 5-hydroxymethylfurfural and morroniside. Loganin belongs to iridoid glycoside, which is also one of the index components in quality control of crude Fructus Corni and processed products in the 2015 edition of Chinese Pharmacopoeia. Loganin exhibits a high content in Fructus Corni and stable chemical property, in addition to easy accessibility. In comparison, several other 


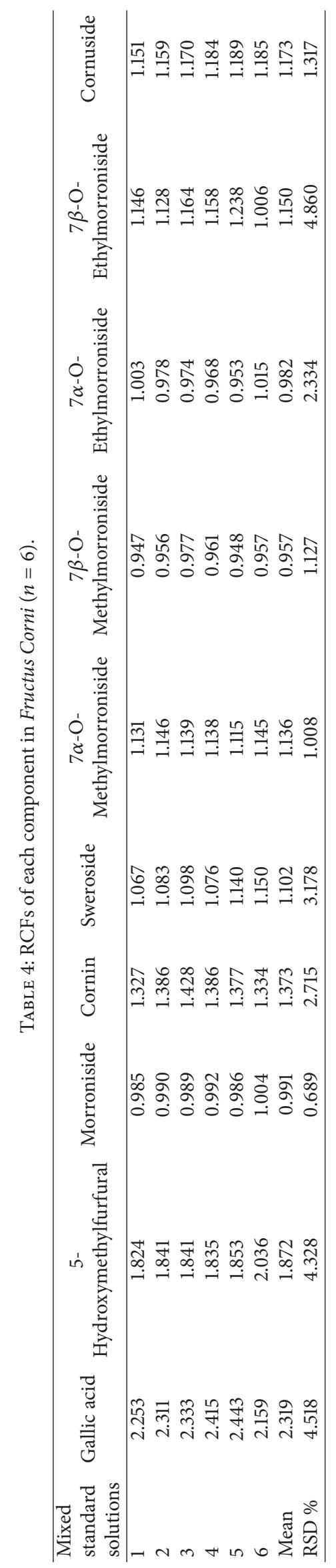




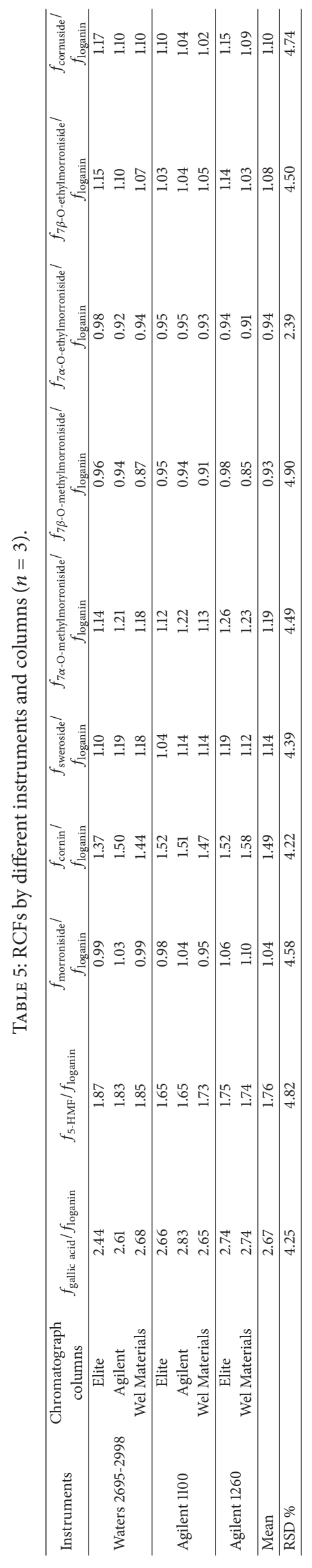




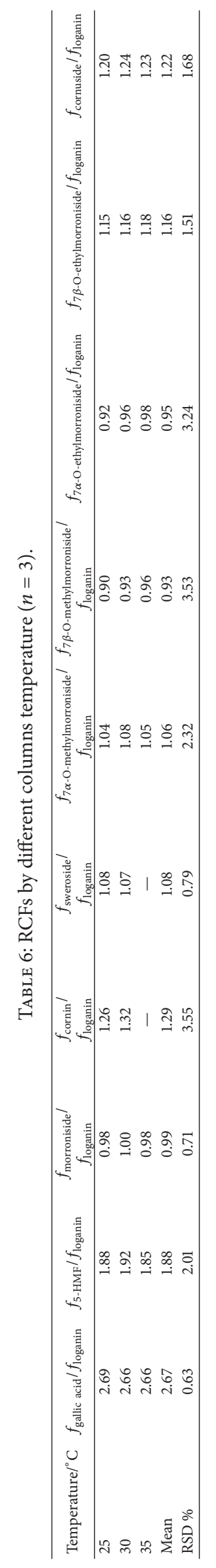




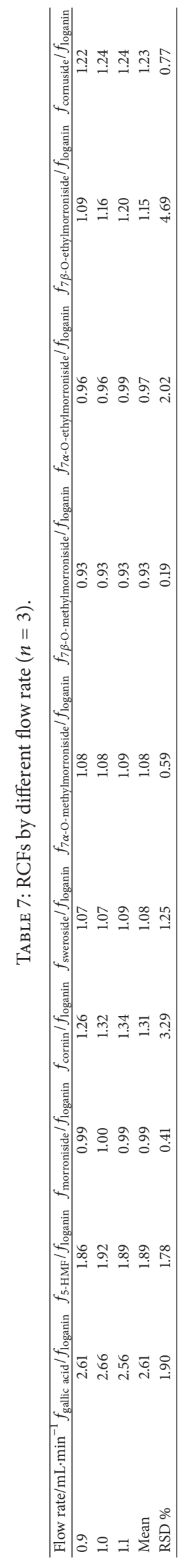




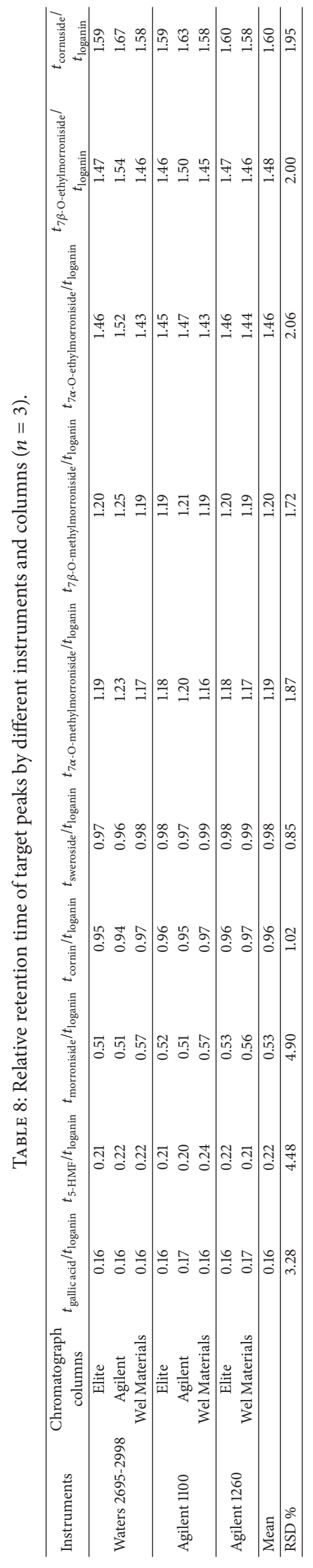




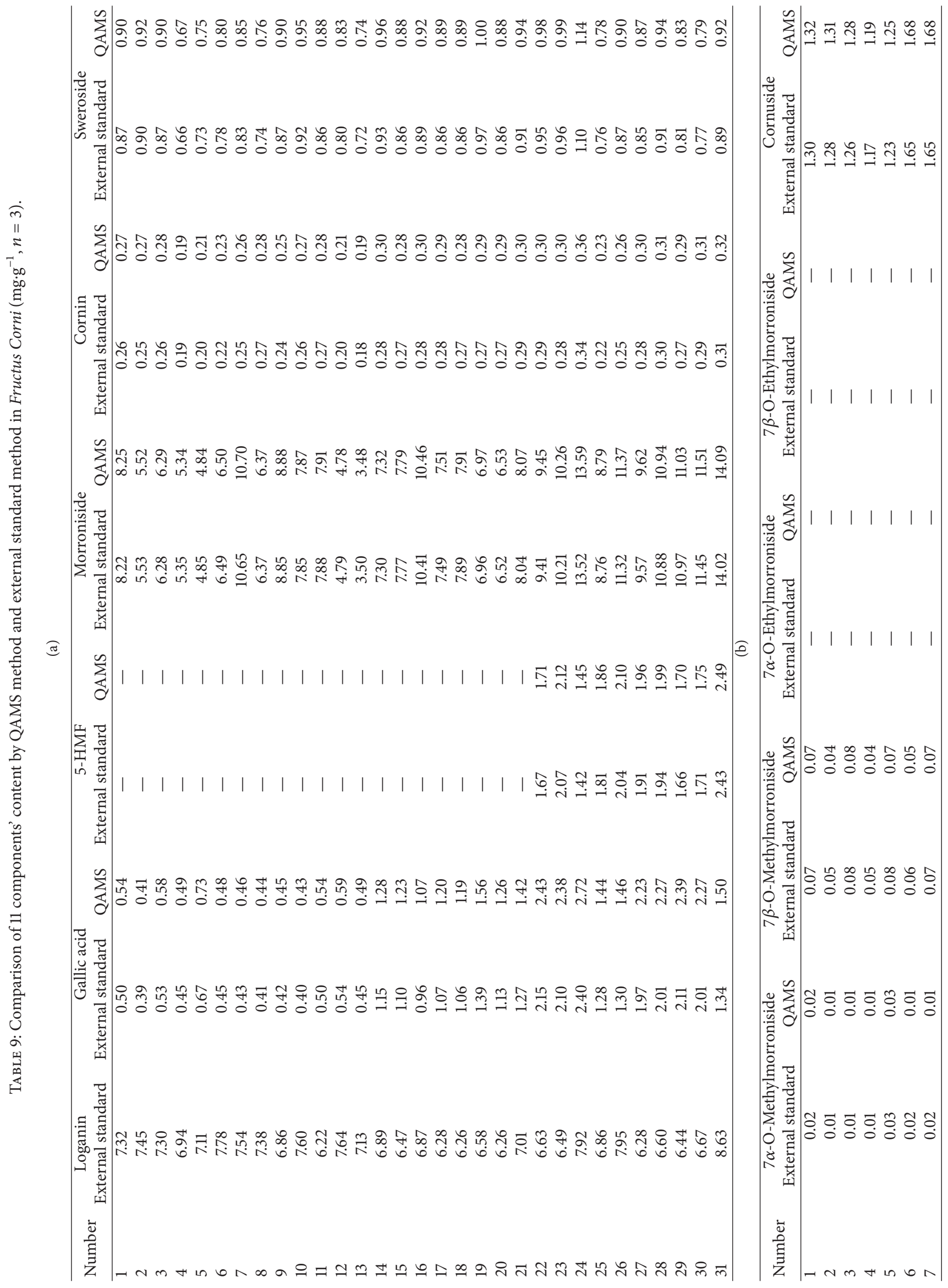




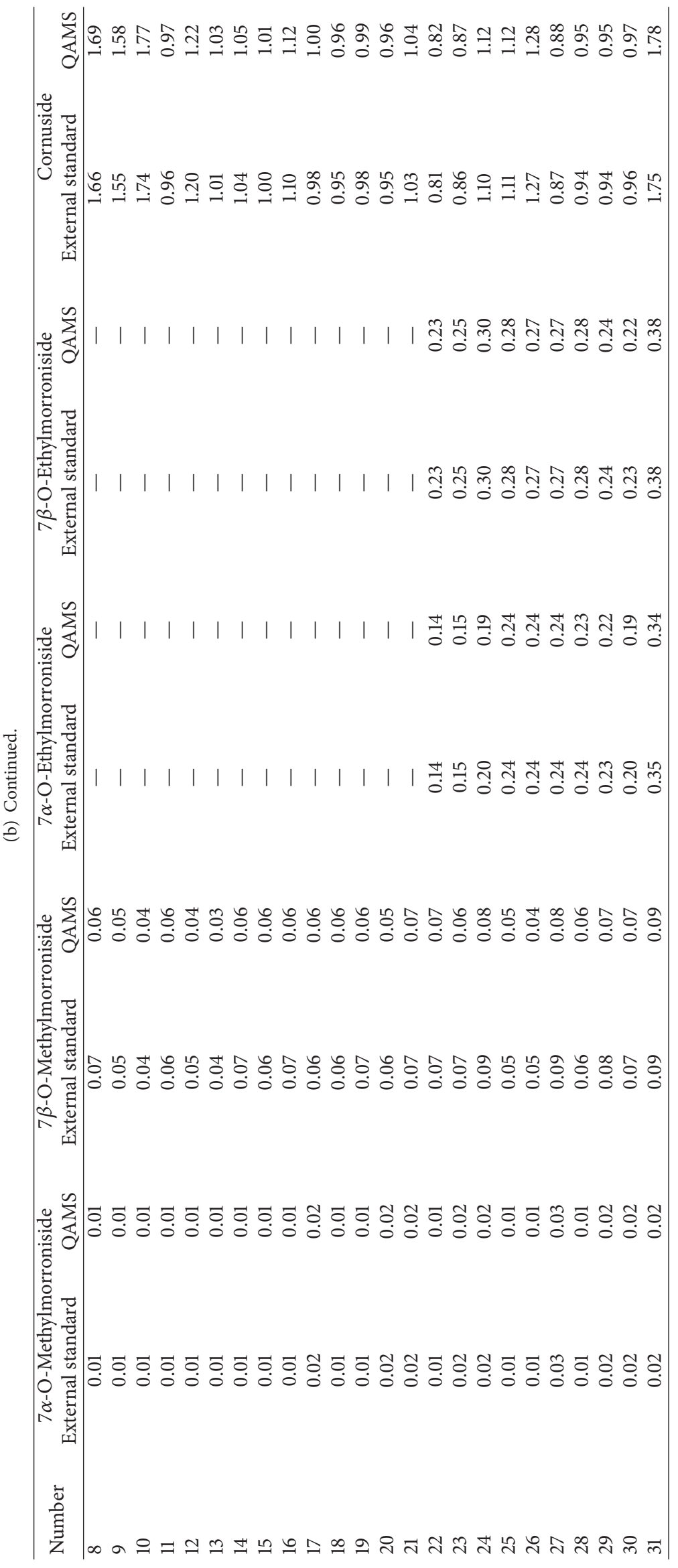


iridoid glycoside components were relatively expensive, and some iridoid isomers (e.g., $7 \alpha$-O-methylmorroniside, $7 \beta$ O-methylmorroniside, $7 \alpha$-O-ethylmorroniside, and $7 \beta$-Oethylmorroniside) were not readily available in the market. Taking these into account, loganin was selected as the internal standard in the development of the QAMS method.

When the QAMS method was applied for quantitative determination, only the internal content needs to be prepared with standard solutions, and the peaks of other components could not be directly confirmed. Therefore, how to locate the chromatographic locations of other target components accurately was one of the key steps for achieving the QAMS determination. In our study, we used the relative retention time between loganin and other components to locate their chromatographic peaks. Previous studies have proved that this method was simple and accurate, especially when it was used for those compounds featuring similar chemical properties [18]. As shown in Table 8, the relative retention times of all the compounds were below $5 \%$, which indicated that this method can locate chromatographic peaks accurately.

Efficient separation of the numerous target components of Fructus Corni by HPLC proved challenging, and currently there were few reports for reference, which largely suffered from narrow coverage and poor repeatability. To overcome this difficult issue, in this study, several different mobile phase systems were therefore tried, including methanolwater, methanol-water containing phosphoric acid or formic acid, acetonitrile-water, and acetonitrile-water containing phosphoric acid or formic acid, with different gradient elution system [14-17, 19]. The results showed acetonitrilewater containing $0.05 \%$ formic acid system produced good separation and repeatability. In contrast, other mobile phase systems have many problems, such that the target components could not be separated at the same time, and detection time was too long. It could improve the peak shape and separation effect, prevent the peak tailing, and make the peak sharp and symmetrical. In this paper, this method shows good separation of eleven compounds in 50 mins by the HPLC system, which had greatly shortened the detection time and reflected the convenience and feasibility of the QAMS method. To inspect the influence of different flow rate on RCF values, we set up three different flow velocities: $0.9 \mathrm{~mL} \cdot \mathrm{min}^{-1}, 1.0 \mathrm{~mL} \cdot \mathrm{min}^{-1}$, and $1.1 \mathrm{~mL} \cdot \mathrm{min}^{-1}$. The results were shown in Table 7; RSD values were all less than $5 \%$. To inspect the influence of different columns temperature on RCFs values, we set the temperature to $25^{\circ} \mathrm{C}$ and $30^{\circ} \mathrm{C}$, and each component could be separated; the RSD value was below $5 \%$. But when the columns temperature was $35^{\circ} \mathrm{C}$, only sweroside and cornin could not be separated. In addition, the experiment was varied on three different instruments (Waters 2695-2998, Agilent 1100, and Agilent 1260) and three different columns (Elite $\mathrm{C}_{18}$, Agilent $\mathrm{C}_{18}$, and Wel Materials $\mathrm{C}_{18}$ ) to investigate the influence of different instruments and columns on RCF values. As showed, in addition the Agilent $\mathrm{C}_{18}(4.6 \mathrm{~mm} \times 250 \mathrm{~mm}, 5 \mu \mathrm{m})$ column could not be used in Agilent 1260 (sweroside and cornin could not be separated completely); the other instruments and columns had good RSD values. Therefore, in order to ensure the accuracy of the RCFs of components by QAMS methods, we should prescribe the type of columns, HPLC's liquid phase system, or columns temperature, so that we could obtain satisfactory results.

\section{Conclusion}

In this paper, we determined the content of components by external standard method at first; the QAMS method was used to calculate their contents, and we compared the two methods to verify the accuracy of QAMS method for quality control of Fructus Corni and its processed products. The results showed that there was no significant difference between the two methods. The QAMS method may provide a convenient, fast, and accurate approach in the quality control of traditional Chinese medicine.

\section{Competing Interests}

The authors declare no conflict of interests.

\section{Acknowledgments}

This work was supported by Jiangsu Traditional Chinese Medicine Administration Project (no. FY201503) and Jiangsu Province Social Development Project (no. BE2009696).

\section{References}

[1] National Pharmacopoeia Committee, Chinese Pharmacopoeia, Part 1, China Medical Science Press, 2015.

[2] S. F. Wang, X. G. Chen, Z. De Hu, and Y. Ju, "Analysis of three effective components in Fructus corni and its preparations by micellar electrokinetic capillary chromatography," Biomedical Chromatography, vol. 17, no. 5, pp. 306-311, 2003.

[3] R.-Q. Yao, L. Zhang, W. Wang, and L. Li, "Cornel iridoid glycoside promotes neurogenesis and angiogenesis and improves neurological function after focal cerebral ischemia in rats," Brain Research Bulletin, vol. 79, no. 1, pp. 69-76, 2009.

[4] N. Yamabe, J. S. Noh, C. H. Park et al., "Evaluation of loganin, iridoid glycoside from Corni Fructus, on hepatic and renal glucolipotoxicity and inflammation in type 2 diabetic $d b / d b$ mice," European Journal of Pharmacology, vol. 648, no. 1-3, pp. 179-187, 2010.

[5] G. Cao, H. Cai, Y. Zhang, X. Cong, C. Zhang, and B. Cai, "Identification of metabolites of crude and processed Fructus Corni in rats by microdialysis sampling coupled with electrospray ionization linear quadrupole ion trap mass spectrometry," Journal of Pharmaceutical and Biomedical Analysis, vol. 56, no. 1, pp. 118-125, 2011.

[6] X. Ding, M.-Y. Wang, Y.-X. Yao, G.-Y. Li, and B.-C. Cai, "Protective effect of 5-hydroxymethylfurfural derived from processed Fructus Corni on human hepatocyte LO2 injured by hydrogen peroxide and its mechanism," Journal of Ethnopharmacology, vol. 128, no. 2, pp. 373-376, 2010.

[7] C. Pal, S. Bindu, S. Dey et al., "Gallic acid prevents nonsteroidal anti-inflammatory drug-induced gastropathy in rat by blocking oxidative stress and apoptosis," Free Radical Biology and Medicine, vol. 49, no. 2, pp. 258-267, 2010.

[8] W. Du, H. Cai, M. Wang, X. Ding, H. Yang, and B. Cai, "Simultaneous determination of six active components in crude and processed Fructus Corni by high performance liquid 
chromatography," Journal of Pharmaceutical and Biomedical Analysis, vol. 48, no. 1, pp. 194-197, 2008.

[9] H. Cai, G. Cao, and B. Cai, "Rapid simultaneous identification and determination of the multiple compounds in crude Fructus Corni and its processed products by HPLC-MS/MS with multiple reaction monitoring mode," Pharmaceutical Biology, vol. 51, no. 3, pp. 273-278, 2013.

[10] H.-Y. Zhou, P.-P. Yang, X.-D. Cong, C.-R. Zhang, and B.-C. Cai, "Comparative study on decoction and dissolution of crude and processed corni fructus," Zhongguo Zhong Yao Za Zhi, vol. 38, no. 22, pp. 3888-3892, 2013.

[11] T.-L. Lu, S.-M. Shi, B.-C. Cai, H.-Z. Liu, Y.-M. Chi, and Y. Zhou, "Advances in studies on multi-component determination of Chinese materia medica by QAMS," Chinese Traditional and Herbal Drugs, vol. 43, no. 12, pp. 2525-2529, 2012.

[12] J. Sun, Z. Z. Jiang, R. Q. Yan et al., "Quality evaluation of Astragali Radix products by quantitative analysis of multicomponents by single marker," Chinese Herbal Medicines, vol. 5, no. 4, pp. 272-279, 2013.

[13] L. T. Zhang, Y. Z. fang, D. Y. Feng et al., "Rencent development and prospect of Cornus officinalis," Chinese Traditional and Herbal Drugs, vol. 35, no. 8, pp. 952-955, 2004.

[14] S. Guo, J.-A. Duan, S.-Q. Zhu, and D.-W. Qian, "Establishment of quality standard for Corni Fructus based on the multiple bioactive constituents," Zhongguo Zhongyao Zazhi, vol. 40, no. 15, pp. 3017-3021, 2015.

[15] X. B. Gao, L. Sun, S. Y. Qiao et al., "HPLC fingerprint of Corni Fructus Praeparata Cum Melle," International Journal of Pharmaceutics, vol. 39, no. 4, pp. 330-336, 2012.

[16] N. Yang, Y. N. Liu, J. F. Kang et al., "Optimization of the HPLC fingerprint for Cornus officinalis," Chinese Journal of Pharmaceutical Analysis, vol. 34, no. 11, pp. 1989-1994, 2014.

[17] W.-F. Du, X.-J. Liang, H. Cai, B.-C. Cai, Y. Zhang, and X.-D. Cong, "Study on fingerprint of crude and processed Cornus officinalis by HPLC," Journal of Chinese Pharmaceutical Sciences, vol. 44, no. 14, pp. 1059-1062, 2009.

[18] Z.-L. Luo, F. Qiu, R.-W. Wei et al., "Application of relative correction factor in multi-index determination of Chinese materia medica," Chinese Traditional and Herbal Drugs, vol. 43, no. 7, pp. 1448-1452, 2012.

[19] Z. R. Chang, J. Li, B. Hao et al., "Analysis of characteristic chemical components in crude and processed Fructus Corni," Chinese Journal of Pharmaceutical Analysis, vol. 35, no. 2, pp. 338-343, 2015. 

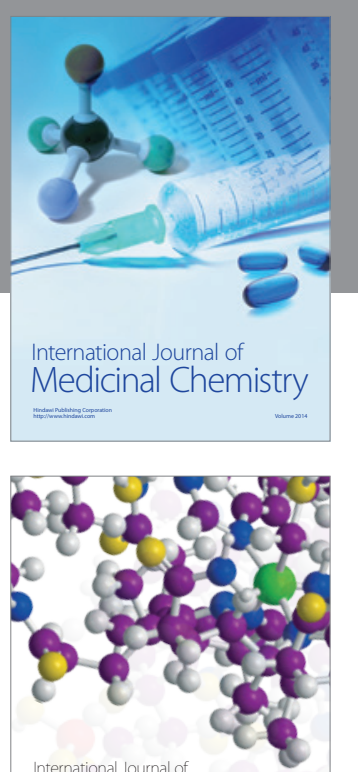

Carbohydrate Chemistry

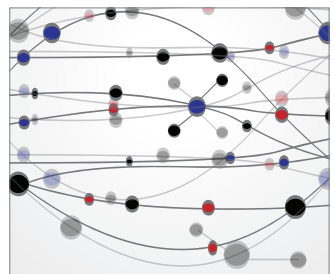

The Scientific World Journal
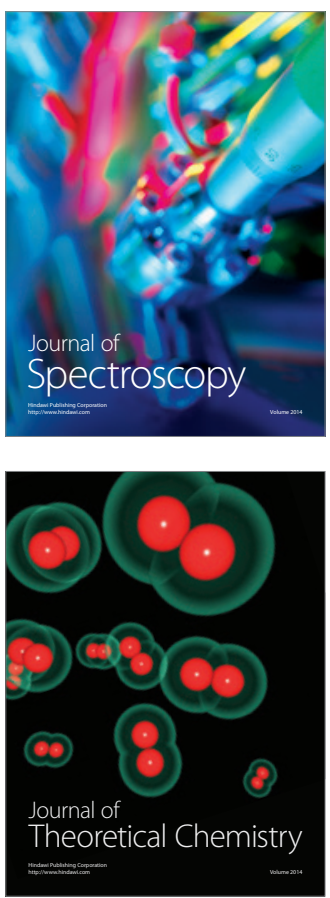
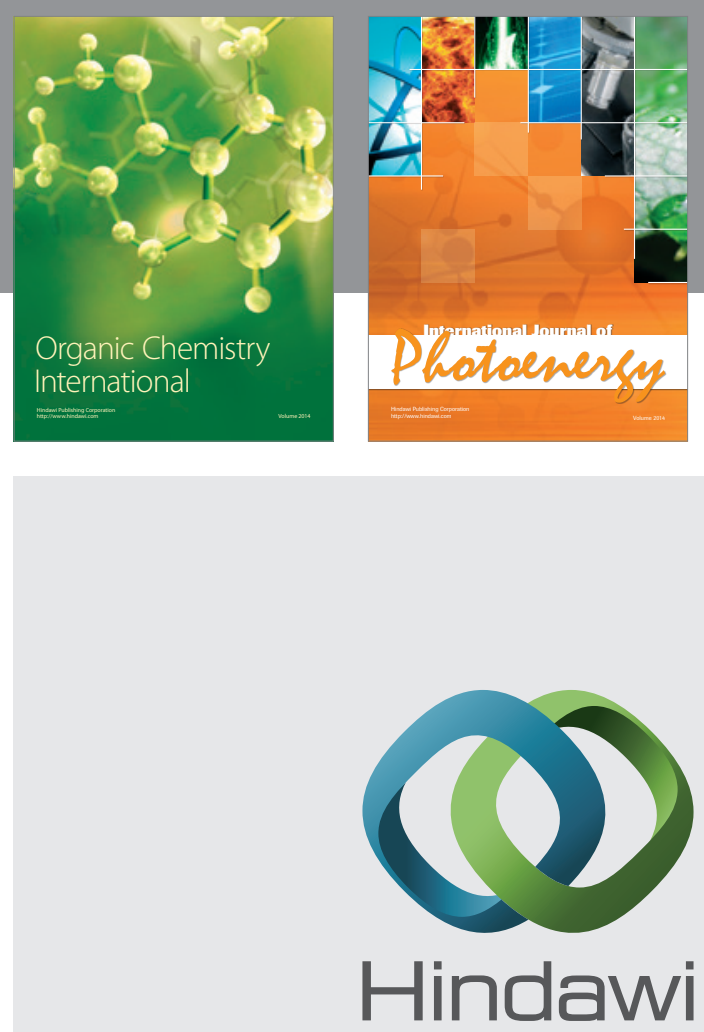

Submit your manuscripts at

http://www.hindawi.com

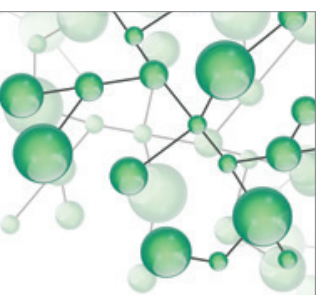

International Journal of

Inorganic Chemistry

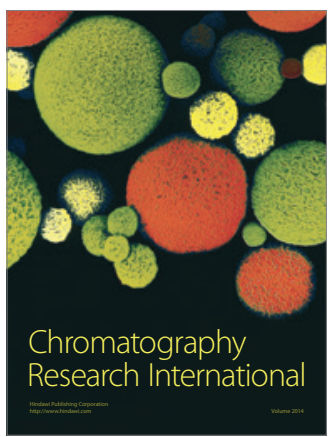

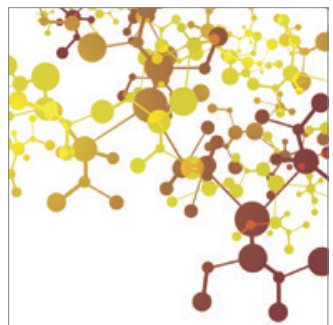

Applied Chemistry
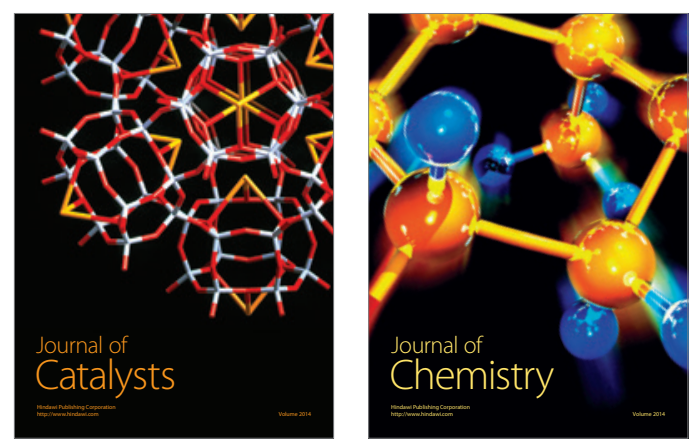
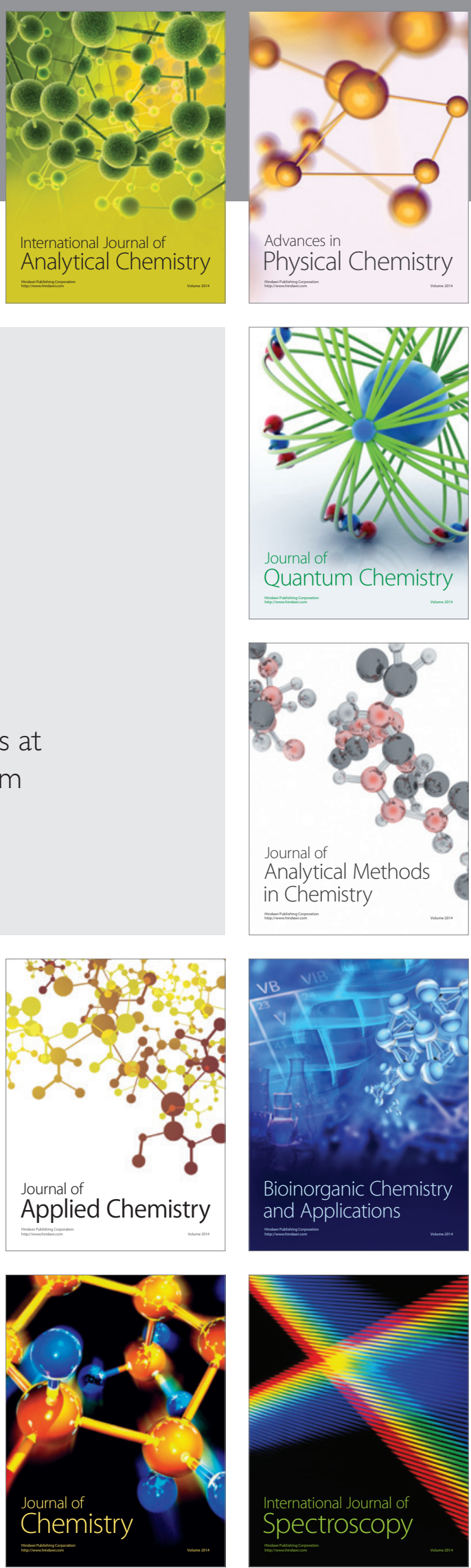